\title{
Shake-The-Box particle tracking with variable time-steps in flows with high velocity range (VT-STB)
}

\author{
D. Schanz ${ }^{1, *}$, M. Novara ${ }^{1}$, A. Schröder ${ }^{1}$ \\ 1: German Aerospace Center (DLR), Inst. of Aerodynamics and Flow Technology, Göttingen, Germany \\ *daniel.schanz@dlr.de
}

\begin{abstract}
We present a novel evaluation mode for Lagrangian Particle Tracking methods in general, applied to the Shake-TheBox method specifically. The aim is to attain high levels of accuracy and a removal of false ('ghost') tracks in flow situations, where significant amounts of particles show small relative movement with respect to each other in consecutive time-steps.

An iterative approach using variable time separations is employed, which starts by tracking particles at high timeseparations, followed by an iterative reduction of time separation, while feeding the particle tracked within the previous iterations. The process allows for applying tracking parameters fine-tuned to the different flow regimes tracked within each iteration.

Experimental validation was performed using a dataset on impinging jet flow, created in collaboration with the School of Mechanical Engineering of Pusan National University. Evaluation of this flow with high velocity range shows distinct advantages in reduction of ghost tracks and in tracking accuracy.
\end{abstract}

\section{Introduction}

Lagrangian Tracking of tracer Particles (LPT) has been conducted since more than three decades (often termed as 3D PTV; Nishino et al. 1989; Maas et al. 1993; Malik et al. 1993). Typically, particle positions were determined using triangulation (Maas et al. 1993); the extraction of particle trajectories was carried out as a secondary step by searching for connected paths within the series of particle clouds reconstructed from each time-step individually (see e.g. Ouellette et al. 2006). Experiments were typically limited to low tracer particle concentrations, as with increasing particle image density more and more ambiguities are created by the triangulation step (creation of 'ghost particles' at positions where no true particle is present) and the subsequent tracking step (creation of 'ghost tracks'). The particle image density $N_{I}$ was typically limited to approx. 0.005 particles per pixel (ppp, meaning that each particle image is described by 200 camera pixels on average) and results were limited to averaged quantities.

The introduction of Iterative Particle Reconstruction (IPR, Wieneke 2013, Jahn et al. 2021) was a first major step towards particle position determination in concentrations that allow resolving unsteady flow dynamics by means of the analysis of individual snapshots. This method represents an iterative approach to the triangulation process with intermediate position optimization and ghost particle removal, allowing to limit the occurrence of ghost particles.

The limitations in particle image density for LPT were ultimately pushed by the 'Shake-The-Box' (STB) algorithm (Schanz et al. 2016), which utilizes a comprehensive prediction of the particle cloud in future time-steps in order to seize temporal information given by the physical consistence and coherence of each particle trajectory. The errors introduced by the prediction can be corrected using an image matching scheme ('shaking', Wieneke 2013, Jahn et al. 2021), thus distinctly facilitating the reconstruction process of each individual time-step, as only particles that are not yet tracked need to be reconstructed using IPR. In its converged phase, STB can be regarded as an integrated process, where the determination of particle trajectories and the reconstruction of the 3D particle field are conducted quasisimultaneously for the large majority of particles.

Applying the STB algorithm, particles can be tracked in large numbers, with a nearly complete suppression of ghost particles. Using synthetic data, images with $N_{I}$ up to $0.2 \mathrm{ppp}$ could be processed (see Digital Content of Raffel et al. 2018), for experimental data particle image densities of up to $N_{I}=0.15 \mathrm{ppp}$ were reached at well-controlled experimental conditions (Huhn et al. 2017, Bosbach et al. 2019).

The ability of the algorithm to discern real particles from ghost particles is largely stemming from the observation that the vast majority of real particles follow reasonably straight trajectories (the change of acceleration is small between time-steps), while ghost particles are generated in quasi-random locations all over the measurement volume. In order 
to register a new track, STB requires the presence of a particle in four consecutive time-steps with limited total acceleration. Therefore, the generation of a ghost track from at least four ghost particles is unlikely in most flow scenarios. However, this assumption only holds if the particle field exhibits a certain amount of movement of the particles relative to each other, such that the ambiguities caused by a certain particle image distribution 'de-correlate' within few time-steps. In many flows this assumption holds, however flows with a high velocity range can exhibit regions in space with only very little movement of the particles from image to image. Jet flows for example typically show a steep gradient of the velocities in the jet center in relation to the outer flow. As the time separation between successive frames has to be chosen such that the fast particles within the jet can be reliably tracked (limiting their movements to e.g. 10-20 pixels), particles in the surrounding flow might move only a fraction of a pixel in the same time. Other examples would be separated or cavity flows, or even boundary layers with particles moving in the bulk at significant velocity but low relative shift due to small velocity gradients. In such a situation a ghost particle, generated by particles from the outer cloud, might be present in several successive frames, therefore potentially generating a ghost track.

Another potentially problematic effect of slowly moving particle clouds is that situations of overlapping particle images are retained over several time-steps. When the images of two particles overlap within a certain camera, the position accuracy of the involved particles is compromised. We will call the local overlapping of two or more particle images a Particle Image Cluster (PIC). In case the PIC corresponds approximately to the sum of the single particle images, the shaking process is able to efficiently resolve the overlap situation. This condition is typically met in synthetically generated particle images and selected experiments, e.g. imaging Helium-filled soap bubbles (HFSB) which are illuminated by an incoherent light source (typically LEDs).

However, for most experimental conditions, the presence of several particles in the same line-of-sight of a camera influences the imaging of each of these particles. For large tracer particles (e.g. in water) a particle close to the camera might cause shadowing of particles located further away. In case coherent laser light is used for illumination, the light scattered on the involved particles might interfere and cause e.g. a speckle pattern. In any of these cases, the PIC will not be the sum of the single particle images of the involved particles and the shaking process will be compromised, resulting in a $3 \mathrm{D}$ displacement of the tracked particle in the time-step where the overlap situation occurs. If such a situation is limited to a single time-step $t_{n}$, STB is typically stable enough to still predict a location at $t_{n+1}$ that is accurate enough for the shaking process to converge to the true position. However, if an overlap situation is sustained over several time-steps, the bias in particle position will have a stronger influence on the position prediction (the prediction uses a Wiener-filter over the last 10 time-steps with increasing weights towards the current time-step). Therefore the risk increases of predicting a particle position with an error that cannot be recovered by the shaking process (the original particle image and the back-projected image of the particle at the assumed position has to overlap on all camera projections). In such a case, several things can happen: (a) ) if the location backprojects to the same coherently moving PIC, a position bias effect of the residual minimization scheme can occur; (b) if the back-projected location lies outside the PIC, the intensity of the predicted particle either could be quickly decreased by the shaking process, as the images do not support the predicted location or (c) the particle still could find sufficient energy on the images of one or more cameras, thereby remaining above the intensity limit despite a corrupted 3D position.

A phenomenon that can sometimes be observed is that such a rogue particle takes the energy, required to survive over multiple time-steps from one specific camera, in which a particle image is moving only slowly. In this case, the particle trajectory is diverted into a direction following the line-of-sight of this camera. Such line-of-sight tracks were quite common in early STB evaluations, before the omission of the brightest camera during the intensity update was introduced (see Section 2.2.3 in Schanz et al. 2016). This measure avoids such effects in most flows, however when a whole region of the particle field is barely moving, the effect can still occur.

\section{Variable Time-step Particle Tracking using the Shake-The-Box method (VT-STB)}

From the thoughts above we can conclude that a slowly moving particle field is detrimental to the tracking process in multiple ways. In this work we propose an extension to the standard STB evaluation, which applies multiple iterations of particle tracking with variable time-separation. The concept is applicable to other tracking methods as well. The idea is to start the evaluation with a time separation such that the slowest particles of the flow can be optimally tracked. From there, the time-separation is iteratively reduced, tracking faster and faster particles with every iteration. Finally, the original time-separation of the recording is reached, where only the fastest particles - whose velocity initially determined the recording rate - remain to be tracked. The involved steps are detailed in the following 
1. Choose a suitable temporal $\Delta_{s, i=1}$ such that the slowest particles move at least one particle image diameter from image to image, where $\Delta_{s, i=1}$ indicates the number of snapshots omitted from the original time-series for this first iteration (e.g. $\Delta_{s, i=1}=50$ : processing of every 50th snapshot)

2. Perform particle tracking on the selected subset of snapshots.

3. Filter the gained trajectories using a fitting method with a suitable kernel-size. In our case TrackFit (Gesemann et al. 2016) was used with an adapted cross-over frequency.

4. Choose a finer temporal resolution $\Delta_{s, i+1}<\Delta_{s, i}$ for the next iteration.

5. Interpolate the filtered trajectories from $\Delta_{s, i}$ to the time-steps given by $\Delta_{s, i+1}$

6. Perform particle tracking on the snapshots given by $\Delta_{s, i+1}$, while feeding the interpolated particle clouds from the previous iteration as a partial pre-solution to the reconstruction of each snapshot

7. Repeat steps from 3 to 6 until $\Delta_{S, i+N}=1$ is reached.

By this approach, the particles from different velocity regimes are always tracked with an optimal time separation. In the process, a few details have to be considered, but also a number of further advantages arise.

- In case a particle is transported from a slowly moving region into a region of higher activity, it's track might be lost within the current iteration as the time-separation becomes too large for the now higher velocities. In order to ensure a reliable, uninterrupted tracking in such situations, the tracking is continued within the next iteration: When within iteration $i$ the end of a track copied from iteration $i-1$ is reached, the track is added to the list of predicted particles and will from there on be continued by the normal STB procedure of iteration $i$. For this purpose it is beneficial to delete the last one or two time-steps from the end of all tracks that were lost in-between the time-series, as the trajectories might already have been compromised a few time-steps before the particle ultimately fell below the intensity threshold and the track was ended.

- The tracking within each iteration can optionally be conducted using a two-pass approach (first walking forwards in time, then reversing and elongating the found tracks backwards in time). This approach is recommended as it provides more symmetric tracking results by counter-acting the slow build-up of tracks at the beginning of the time-series (see Schanz et al. 2016 for details).

- In order to avoid small-scale noise effects due to e.g. overlap situations, the shaking process can be deactivated for copied particles at lower values of $\Delta_{s}$. This means that the intensity is still updated, however the particle position remains unchanged for the particles taken from the previous iteration. Please note that this measure represents a filtering effect on slowly moving particles, as only a sub-set of the available time-steps is used for the creation of the filtered trajectory. Therefore the interaction of the chosen filtering and the sampling rate at which shaking of copied particles is turned off has to be characterized and adapted to the flow. An automated procedure for defining these parameters is still under development. However, in general, the possibility to adapt filtering strength to the velocity of the particles is welcome, as in most flows, the dynamics scale with velocity and a stronger filtering can be applied to slow particles.

Lagrangian Particle Tracking using STB can be divided into two different modes, for which separate parameters exist. Some of these can and have to be chosen independently for the different iterations :

\section{Finding new tracks:}

- $\boldsymbol{P}$ : Shift vector applied to a given particle position $\overrightarrow{x_{p}}$, at time-step $i$ for a search of connected particles $p^{\prime}$ in the next time-step. Within the initialization phase, $\mathrm{P}$ is constant over the volume (e.g. $[0,0,0])$; once the tracking system is established, $\mathrm{P}$ is given locally by the averaged velocities of neighboring tracked particles.

- $\quad \boldsymbol{R}$ : Search radius around $P$. All particles $p^{\prime}$ in the cloud of yet untracked particles at time-step $i+l$ are identified that satisfy $\left|\overrightarrow{x_{p}}+P-\overrightarrow{x_{p \prime}}\right|<R$. For every such particle a track candidate is created. The process is repeated in the following time-steps, elongating track candidates in case particles that satisfy the condition are found in each time-step.

- $\boldsymbol{R}_{\text {min }}$ : minimum shift of track. Excludes particles found in the particle cloud of time-step i+1 that are closer than $R_{\min }$ to the previous coordinate (e.g. a particle has to move at least one pixel at $R_{\min }=1$ ).

- $\Delta_{n t}$ : smoothness threshold for new tracks. Once a track candidate reaches a length of four time-steps, a function $F\left(\overrightarrow{x_{n}}\right)$ (typically 2nd order) is fitted to the four coordinates $\overrightarrow{x_{n}}$. A track is assumed to be found in case the average difference of the particle positions to the fitted ones $\Delta_{\overrightarrow{x_{n}}}=\frac{1}{n} \cdot \sum_{n}\left|F\left(\overrightarrow{x_{n}}\right)-\overrightarrow{x_{n}}\right|$ satisfies 
$\Delta_{\overrightarrow{x_{n}}}<\Delta_{n t} . \Delta_{n t}$ is given in pixel; typical values range from 0.2 to 1.5 , depending on the flow. This step can be regarded as limiting the allowed acceleration of the track fragment in relation to a 2 nd order fit. In case a particle is part of several valid track candidates, the one with the lowest $\Delta_{\overrightarrow{x_{n}}}$ is chosen.

\section{Elongating existing tracks:}

- $\Delta_{p r}$ : deviation threshold from prediction. All particles $p$ for which a valid track exists at time-step $i$ are predicted to $i+l$ by fitting and extrapolating a function to the last (up to ten) known time-steps. To this end, a Wiener-filter approach is used with increasing weight towards the current time-step, which is extrapolated to $\mathrm{i}+1$, yielding a coordinate $\overrightarrow{x_{p r}}$. All predicted coordinates are inserted as particles into the IPR process of timestep $i+l$ and are treated by several iteration of the position optimization process (shaking). This way, the errors introduced by the prediction are corrected. In case the error is too large, the particle is either deleted as its intensity is reduced or it randomly walks in 3D-space, following some residuals on the particle images. The parameter $\Delta_{p r}$ defines, how much deviation from the prediction point is allowed for a tracked particle. The one particle $p$ that minimizes $\left|\overrightarrow{x_{p}}-\overrightarrow{x_{p r},}\right|<\Delta_{p r}$ for a given track $t$ is chosen for continuing $t$ in the current time-step. If no such particle exists within the cloud generated by IPR, $t$ is ended.

- $\boldsymbol{T}_{\boldsymbol{O}}$ : Outlier filter. In order to identify and terminate tracks that have deviated from the real particle positions as quickly as possible, an outlier filter is employed, as explained in (Schanz et al. 2016): For each tracked particle, $r m s d_{\vec{v}}=\sqrt{\frac{1}{n} \sum_{n}\left(\vec{v}-\vec{v}_{\text {avg }}\right)}$ signifies the root mean squared deviation of the velocities of the $n$ neighbouring particles with $\vec{v}_{\text {avg }}$ being their average. The current particle $p$ is regarded an outlier if $\left|\vec{v}_{p}-\vec{v}_{a v g}\right|>T_{O} \cdot r m s d_{\vec{v}}$

In a single-iteration evaluation with $\Delta_{S}=1$ the parameters for outlier detection and track identification have to be chosen uniformly and in such a way that particles that exhibit the highest acceleration can be tracked and are not discarded as outliers. For large parts of the volume these values could typically be lowered, which would decrease the likelihood of ghost track creation. With VT-processing this discrimination becomes possible and furthermore is not restricted to pre-defined regions in space, as the method intrinsically determines the regions of low and high flow velocities.

In the next paragraph the VT-STB approach is applied to an experimental dataset, measuring the flow of a small diameter jet impinging on a circular cylinder. The described features and advantages of VT-processing are heavily connected to experimental difficulties, e.g. particle images not summing up perfectly, particle reprojection not perfectly matching the real particle image (despite a proper calibration of the optical transfer function (OTF, Schanz et al. 2013), differences between the perceived particle intensity from the different cameras, and more. As it is difficult to simulate all the mentioned effects accurately it was decided to directly move to the evaluation of experimental data, without a prior test on synthetic data.

\section{Experimental validation}

\subsection{Experimental setup}

The experimental setup is submerged in water within a regular cylindrical 16 -faces glass tank. A jet is generated by a nozzle of diameter $d=3 \mathrm{~mm}$ and is impinging onto a circular cylinder with diameter $d_{C}=52 \mathrm{~mm}$ at an angle of $45^{\circ}$. The velocity of the jet flow was varied, however for the remainder of this work, we will only consider a case with a maximum jet velocity $v_{M A X}$ of $6 \mathrm{~m} / \mathrm{s}$, corresponding to a Reynolds number of 17,875, with Re being defined by $\left(\frac{4 Q}{\pi d^{2}}\right) d / v$, where $\mathrm{Q}$ is the volumetric flow rate and $v$ the kinematic viscosity. The height $H$ of the jet over the cylinder was fixed at $H=4 d$. For a more detailed description, see (Kim et al. 2018), where a comparable setup was employed in air.

The flow impinges onto the cylinder and attaches to the surface due to the Coandă effect. Separation occurs only at the bottom part of the cylinder. The flow is seeded with $40 \mu \mathrm{m}$ Oragasol polyamide particles, which are illuminated by two high-power arrays of white LEDs (Stasicki et al. 2017). The two arrays are positioned on both sides of the water tank, each being collimated by a lens with $\mathrm{f}=1000 \mathrm{~mm}$ focal length (see Fig. 1), illuminating a volume of 

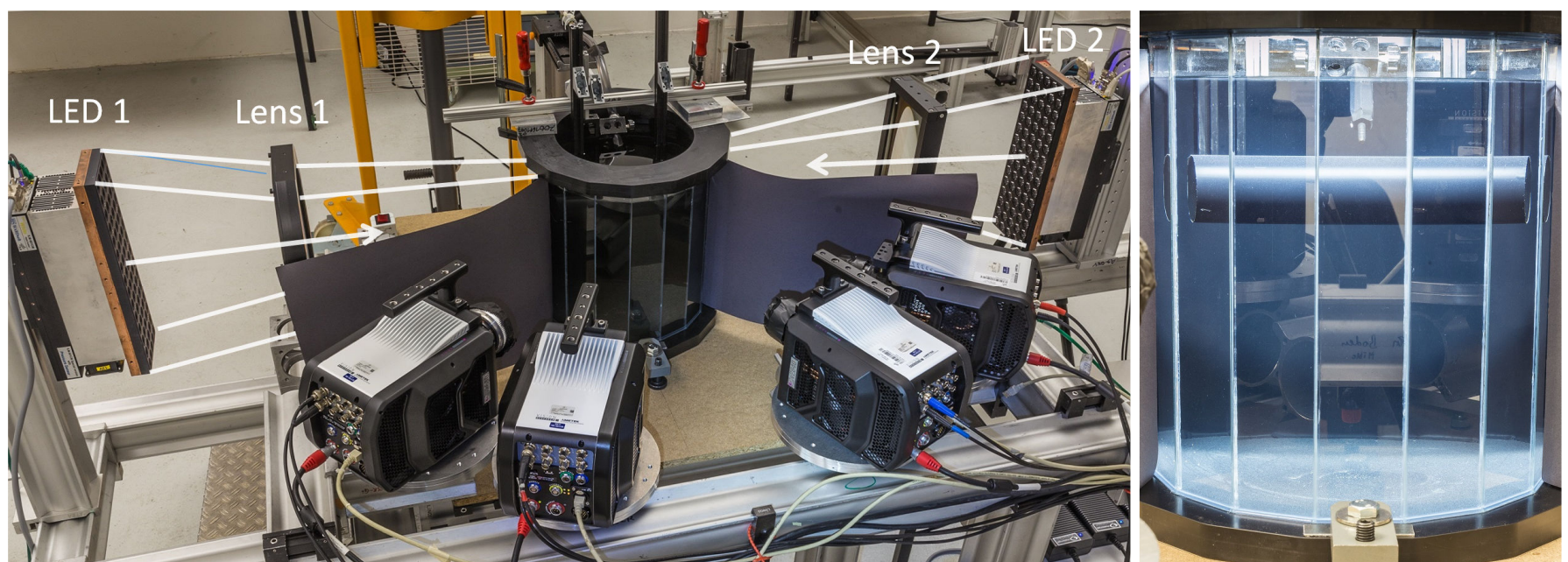

Fig. 1: Setup for the water jet experiment. Left: Four Phantom V2640 cameras viewing into the water tank, which is illuminated from two sides by high-power white LED arrays, each collimated by a $1000 \mathrm{~mm}$ - lens. Right: 16-face water tank with submerged cylinder $\left(\mathrm{d}_{\mathrm{c}}=52 \mathrm{~mm}\right)$ and $\mathrm{d}=3 \mathrm{~mm}$ nozzle at $45^{\circ}$ impinging angle.

approx. $160 \mathrm{~mm}$ height and $50 \mathrm{~mm}$ depth. This measurement region comprises the jet, half of the cylinder in lateral direction and a wake region below the cylinder. See Fig. 2 for a schematical depiction of the measurment region. The measurement volume is observed by a system of four Phantom V2640 high-speed cameras, equipped with $60 \mathrm{~mm}$ Carl Zeiss macro lenses in Scheimpflug mounts. The cameras are operated at $4 \mathrm{kHz}$ repetition rate at a resolution of 1152 $\times 1952 \mathrm{px}$. The volume seen by all cameras comprises approx. $80 \times 160 \times 50 \mathrm{~mm}^{3}$. The internal memory of $144 \mathrm{~GB}$ allows for time-series of 45,407 consecutive time-steps. The camera system is calibrated using a two-plane calibration plate. The geometrical calibration was refined using Volume Self Calibration (Wieneke 2008) and calibration of the optical transfer function (Schanz et al. 2013).

The flow generated by the jet exhibits a very high Velocity Range (VR): particles located within the central axis of the jet move at approx. $6 \mathrm{~m} / \mathrm{s}$, while particles in the outer part of the volume show very little movement, in the order of $0.01 \mathrm{~m} / \mathrm{s}$. The repetition rate was chosen such that the fastest particles can still be tracked reliably, moving not more than approx. 18 pixels between frames. Therefore the particles in the bulk move only approx. 0.03 pixels per timestep, leading to a virtually static particle ditributions in these regions - which are large, due to the small mass-flow of the jet.
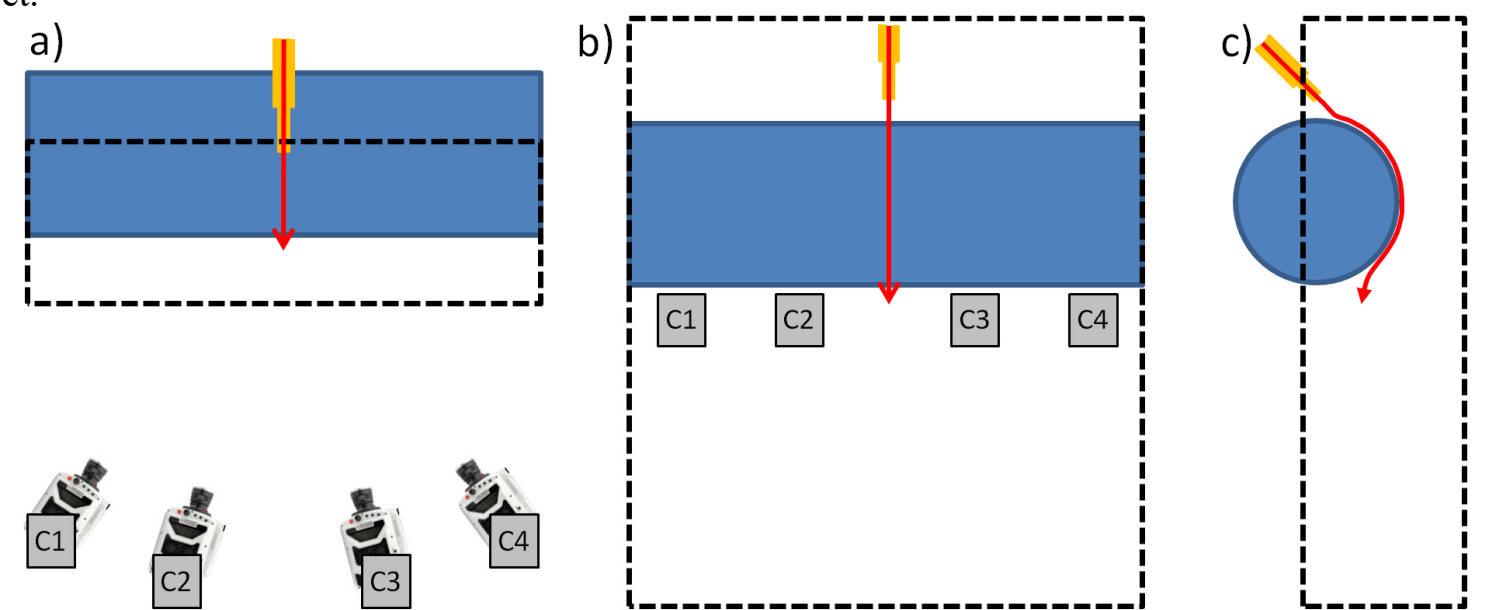

Fig. 2: Schematical experimental setup from top view (a), front view (b) and side view (c). The cylinder is depicted in blue, the nozzle in yellow, the centerline flow in red and the illuminated area as a dashed line. The camera system (C1-C4, not to scale) is setup as an inline-configuration, centered to the illuminated volume.

\subsection{Evaluation}

VT-STB was applied in the following fashion: 5 iterations were performed, starting with a time separation of $\Delta_{s, 1}=$ 79 time-steps (the slowest particle move approx. 2.4 pixel in this interval, corresponding to approx. one particle image diameter). The following iterations feature time separations of $\Delta_{s, i}=[36,14,6,1]$ time-steps. A time-series of 10,000 images was reconstructed. 


\begin{tabular}{|c|c|c|c|c|c|c|c|c|c|}
\hline Iteration & $\boldsymbol{\Delta}_{\boldsymbol{s}}$ & $\boldsymbol{P e a k}$ & $\boldsymbol{R}$ & $\boldsymbol{R}_{\min }$ & $\boldsymbol{\Delta}_{\boldsymbol{n} \boldsymbol{t}}$ & $\boldsymbol{\Delta}_{\boldsymbol{p r}}$ & $\boldsymbol{T}_{\boldsymbol{O}}$ & S. $\boldsymbol{c .}$ & $\boldsymbol{c} \boldsymbol{c}$ \\
\hline VT-STB & & {$[\mathrm{counts}]$} & {$[\mathrm{px}]$} & {$[\mathrm{px}]$} & {$[\mathrm{px}]$} & {$[\mathrm{px}]$} & {$\left[r m s d_{\vec{v}}\right]$} & & \\
\hline 1 & 79 & 13 & 12.6 & 0.0 & 0.3 & 3.5 & 6 & Yes & 0.75 \\
\hline 2 & 36 & 13 & 12.6 & 0.0 & 0.3 & 3.5 & 6 & Yes & 0.6 \\
\hline 3 & 14 & 13 & 12.6 & 0.0 & 0.3 & 3.5 & 7.5 & Yes & 0.5 \\
\hline 4 & 6 & 13 & 12.6 & 1.0 & 0.3 & 3.5 & 9.5 & No & 0.4 \\
\hline 5 & 1 & 20 & 18 & 1.0 & 0.5 & 5.5 & 40 & No & 0.15 \\
\hline STB & & & & & & & & & \\
\hline- & 1 & 20 & 18 & 0.0 & 0.5 & 5.5 & 20 & - & 0.15 \\
\hline
\end{tabular}

Table 1: Parameters used for processing of the different iterations of VT-STB and of normal STB. $\boldsymbol{\Delta}_{\boldsymbol{s}}$ signifies the time-step separation, Peak the required intensity on the image of an identified peak (in counts) for triangulation, $\boldsymbol{R}$ the search radius around the prediction point for new tracks, $\boldsymbol{R}_{\text {min }}$ the required minimum velocity of new tracks, $\boldsymbol{\Delta}_{n t}$ the allowed average deviation from a second order fit for new tracks, $\boldsymbol{\Delta}_{\boldsymbol{p}}$ the search radius around the prediction point for existing tracks, $\boldsymbol{T}_{\boldsymbol{O}}$ the outlier filter parameter for existing track, $\boldsymbol{S}$. $\boldsymbol{c}$. (Shake copied) if the positions of particles from previous iterations are optimized by shaking and $\boldsymbol{c o}$ the crossover-frequency used for filtering the found tracks (see Gesemann et al. 2016).

The parameters applied at each iteration are given in Table 1, as well as the ones used by a standard STB-evaluation, conducted for comparison. For VT-STB processing, parameters need to be set for every iteration. In order to simplify this process, the search radii $\mathrm{R}$ for each time separation of $\Delta_{s}>1$ are set as $R=0.7 \cdot \Delta_{\max }=12.6$ pixel in relation to the assumed maximum displacement of a particle at the original sampling rate $\Delta_{\max }=18$ pixel. Only for the last iteration $R=\Delta_{\max }$ is applied, as this iteration mainly tracks the fast particles in the jet core and the shear layer, where large velocity gradients are present. $\Delta_{n t}$ is set to 0.3 pixels, except for the last Iteration, where $\Delta_{n t}=0.5$ pixels is applied. $\Delta_{p r}$ uses a value of 3.5 pixels for $\Delta_{s}>1$, while 5.5 pixels is used in the last iteration. $R_{\min }$ is set to zero for all iterations, except the last two, where it is assumed that all slowly moving particles have already been identified. This measure prevents the creation of ghost tracks from the residuals of slowly moving particle clouds, as explained above. $T_{O}$ is slowly increased from 6 to 9.5 for iterations 1-4 and then set to 40 for iteration 5 , to account for the high local velocity differences in the shear layer. The normal STB evaluation mainly uses the parameters of the last VTSTB iteration, except that $T_{O}$ is reduced to 20 in order to allow at least some suppression of deviated tracks. All iterations of VR-STB and STB consist of two passes, going once forwards and once backwards in time.
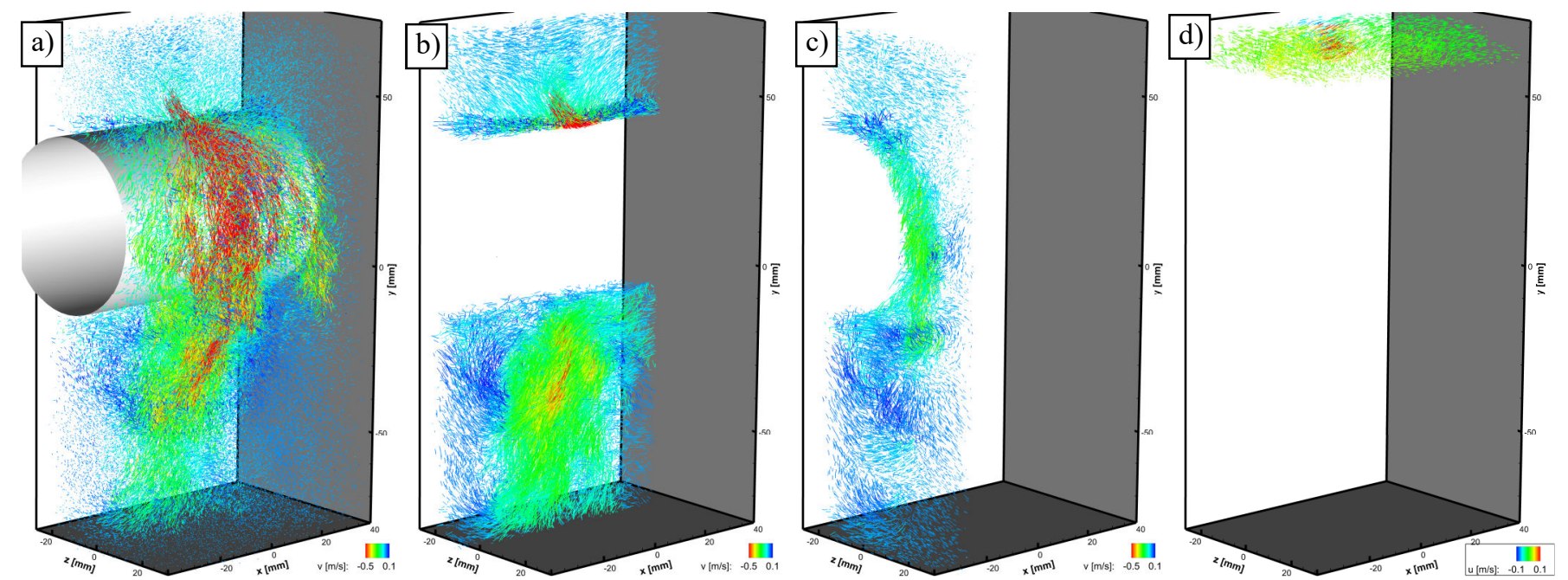

Fig. 3: Instantaneous flowfield as gained with VT-STB. Shown are particle tracks over 50 consecutive time-steps, color-coded by the velocity in downward direction; (b-d): slices used for visualizations in Fig. 5, Fig. 7 and Fig. 8. 

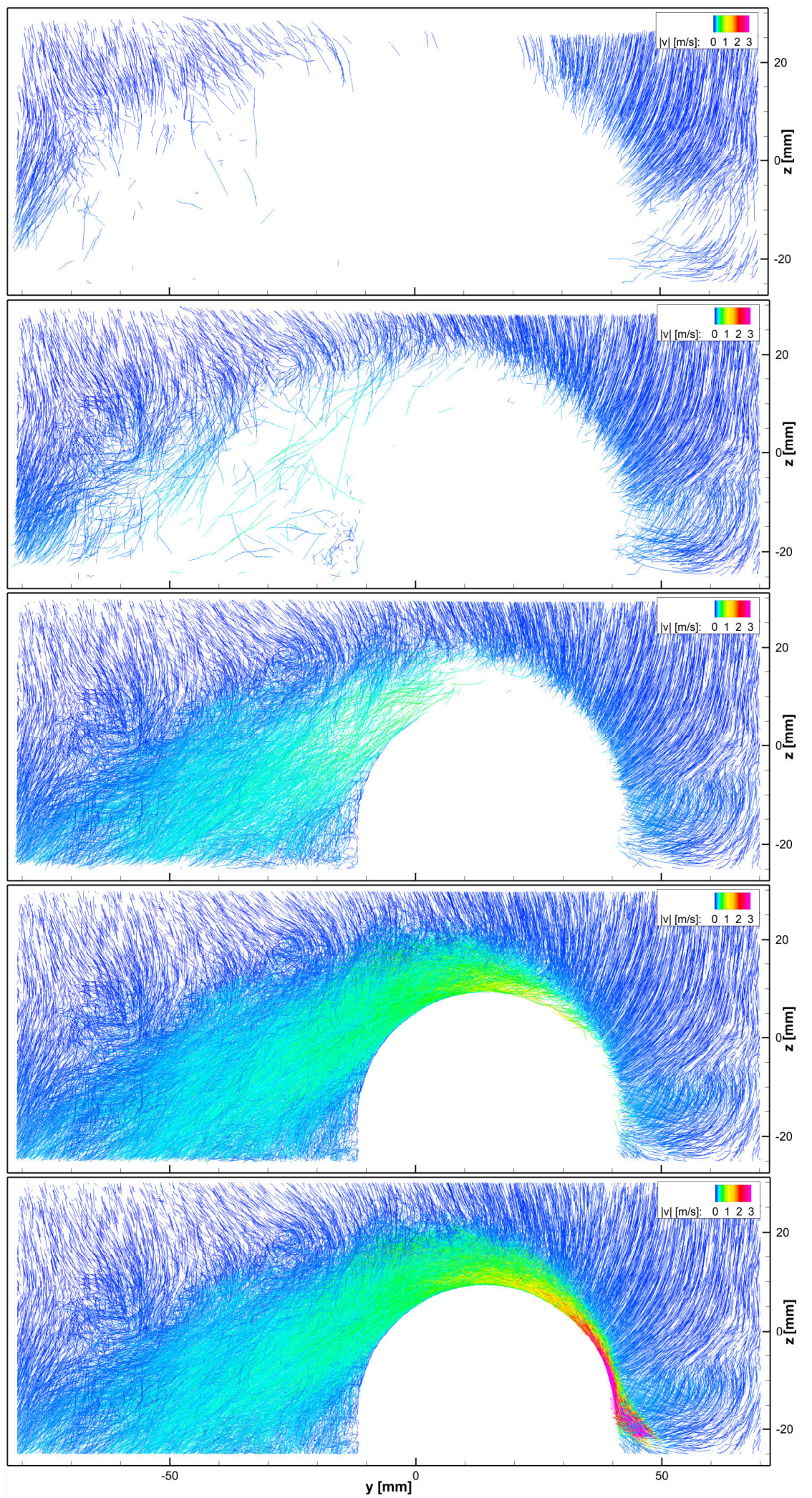

Fig. 4: VT-STB tracking with 5 iterations. From top to bottom: Tracking results in $10 \mathrm{~mm}$ middle slice of coanda water jet for successive iterations. Parameters of each iteration given in Table 1.200 out of 45407 times-steps shown

Fig. 3 (left) shows an instantaneous tracking result of the end-result of VT-STB. The impinging jet flow is captured, which rapidly broadens from the impingement point, creating small-scale flow structures advecting over the cylinder. The fastest particles indeed move at around $6 \mathrm{~m} / \mathrm{s}$, however the color-scale has been adapted for better visibility. The
VT-STB, iteration 1

- Number of particles: approx. 24,500

VT-STB, iteration 2

- $\Delta_{s}=36$ time-steps

- Number of particles: approx. 42,600

VT-STB, iteration 3

- $\Delta_{s}=14$ time-steps

- Number of particles: approx. 65,000

VT-STB, iteration 4

- $\Delta_{s}=6$ time-steps

- Number of particles: approx. 77,300

VT-STB, iteration 5

- $\Delta_{s}=\mathbf{1}$ time-step

- Number of particles: approx. 80,500
- $\Delta_{s}=79$ time-steps

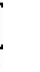


flow separates below the centerline of the cylinder and a wake is formed beneath it. Vast regions of slowly moving particles exist above and below the cylinder.

The operating principle of VT-STB is illustrated in Fig. 4. For each iteration, a 10mm - thick slice of the volume, centered around the nozzle is visualized, showing particle tracks over approx. 500 time-steps (based on the original sampling; the effective length varies slightly due to the different time separations).

The particles tracked at $\Delta_{s, 1}=79$ are located in the entrainment region and below the cylinder, far from the wake of the jet. Approx. 24,500 particles are captured instantaneously. Maximum velocities of approx. $0.12 \mathrm{~m} / \mathrm{s}$ are attained, corresponding to a shift of approx. 30 pixels at the given $\Delta_{S}$ (and 0.37 pixel at $\Delta_{S}=1$ ). This velocity significantly exceeds the initial search radius of $\Delta_{i}=12.6$ pixels, as (a) tracked particles can accelerate and (b) the neighborhood predictor expands the trackable regime to higher velocities with time. Particles accelerating to even higher velocities are typically lost, as the prediction can no longer follow the acceleration at this $\Delta_{S}$.

Feeding the particles tracked in the first iteration to the tracking system of the next one $\left(\Delta_{s, 2}=36\right)$ and performing again two passes of tracking expands the field of tracked particles towards larger velocities. A further number of approx. 18,100 particles is tracked. Here, particles up to $0.3 \mathrm{~m} / \mathrm{s}$ are found. When moving to iteration $3\left(\Delta_{s, 3}=14\right)$, maximum velocities of $0.7 \mathrm{~m} / \mathrm{s}$ can be tracked. Large parts of the wake region below the cylinder appear, with an additional number of 22,400 tracked particles. At $\Delta_{s, 4}=6$ (iteration 4), most of the flow on the cylinder surface can be tracked, singular particles approach velocities of $1.5 \mathrm{~m} / \mathrm{s}$. The tracking system is complemented by 12,300 further particles. From this iteration on, the tracked particles being read from the previous iteration are no longer treated by the shaking process, i.e. their positions remain unchanged; only the intensity is updated. Finally, when $\Delta_{s, 5}=1$ is reached in iteration 5, the remaining fast particles from the jet and it's direct surroundings are tracked. Maximum velocities of $6 \mathrm{~m} / \mathrm{s}$ are seen, as expected. These particles comprise only a small subset of the particles; approx. 3,200 particles are added to the tracking system at each time instant. A total number of just over 80,000 particles is reached.
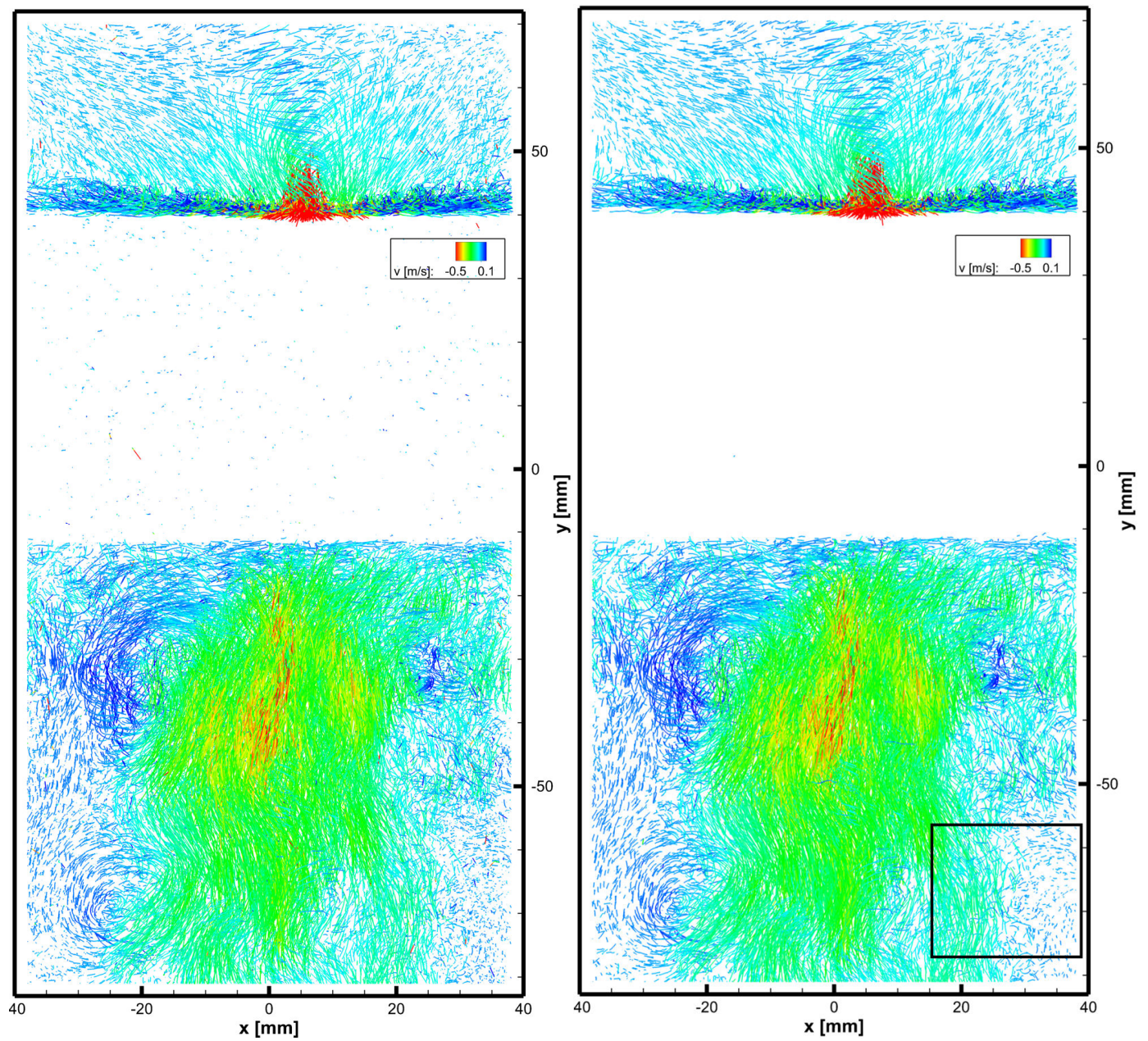

Fig. 5: STB processing (left) vs. VT-STB processing (right), showing tracks for 200 consecutive time-steps in a 10mm-thick slice in the $\mathrm{x}-\mathrm{y}$ plane, located as indicated in Fig. 3 b). Detail view in Fig. 6 marked by rectangle 


\subsection{Comparison to standard evaluation}

In order to assess the benefits of VT-STB over regular processing using $\Delta_{S}=1$, the results are compared to a normal STB evaluation over 1,000 images, with parameters given in Table 1. The normal STB processing finds approx. 1,500 particles less compared to VT-STB, for a total of 79,000 instantaneous particles. Fig. 5 compares the tracking results in a $10 \mathrm{~mm}$-thick x-y plane, with the cylinder being cut by the plane. Shown are particle tracks for 200 consecutive time-steps. Both evaluations correctly capture the vast majority of the tracks, however for STB a relevant amount of tracked particles appears within the cylinder, indicating ghost tracks. For VT-STB this region is nearly completely void. Within the particle clouds, STB shows traces of falsely tracked particles, clearly standing out from the surroundings. This becomes more evident, when focusing on a smaller region (see Fig. 6). While the STB-result is interspersed with both outliers, as well as a sizeable number of tracks with velocities close to zero, appearing in regions with visibly higher flow velocity, the VT-STB results is free of both types of abnormal particle tracks. At the same time, the tracks of slow particles exhibit less noise, as the filtering is carried out on a broader kernel, minimizing the effects of long-lasting overlap situations.

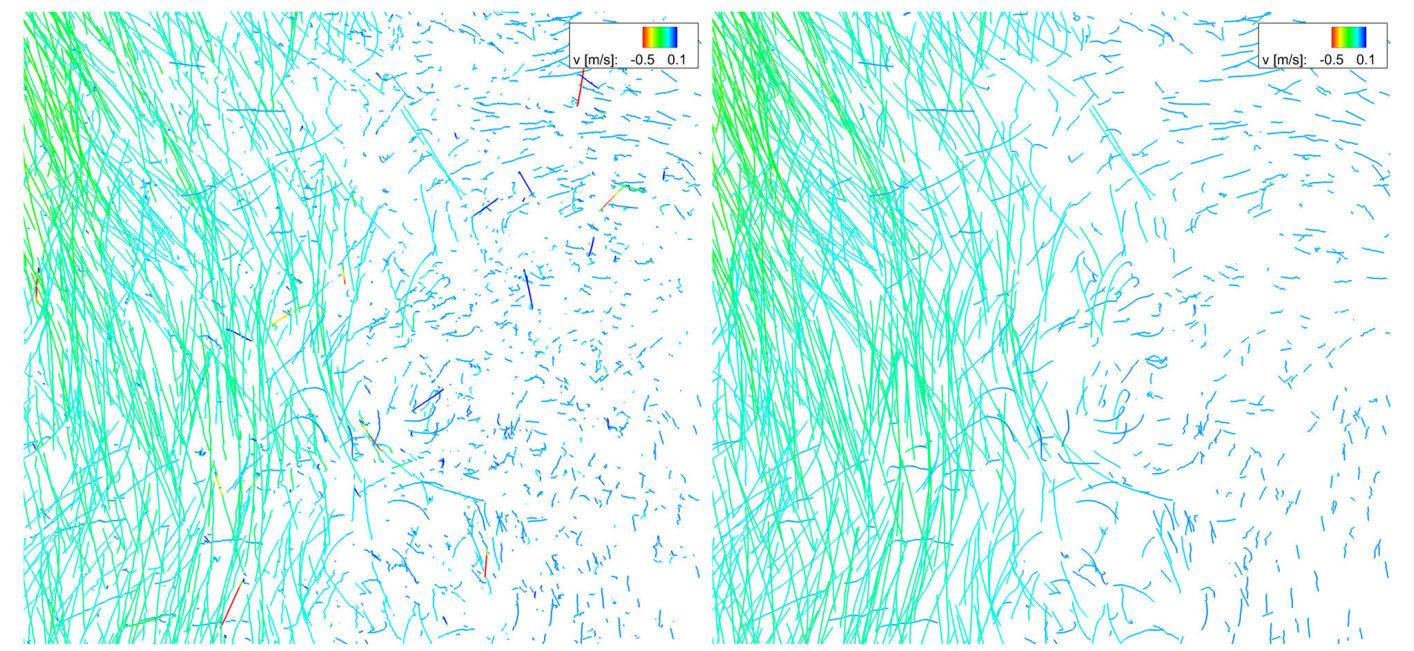

Fig. 6: STB processing (left) vs. VT-STB processing (right). Excerpt from Fig. 5 at marked position
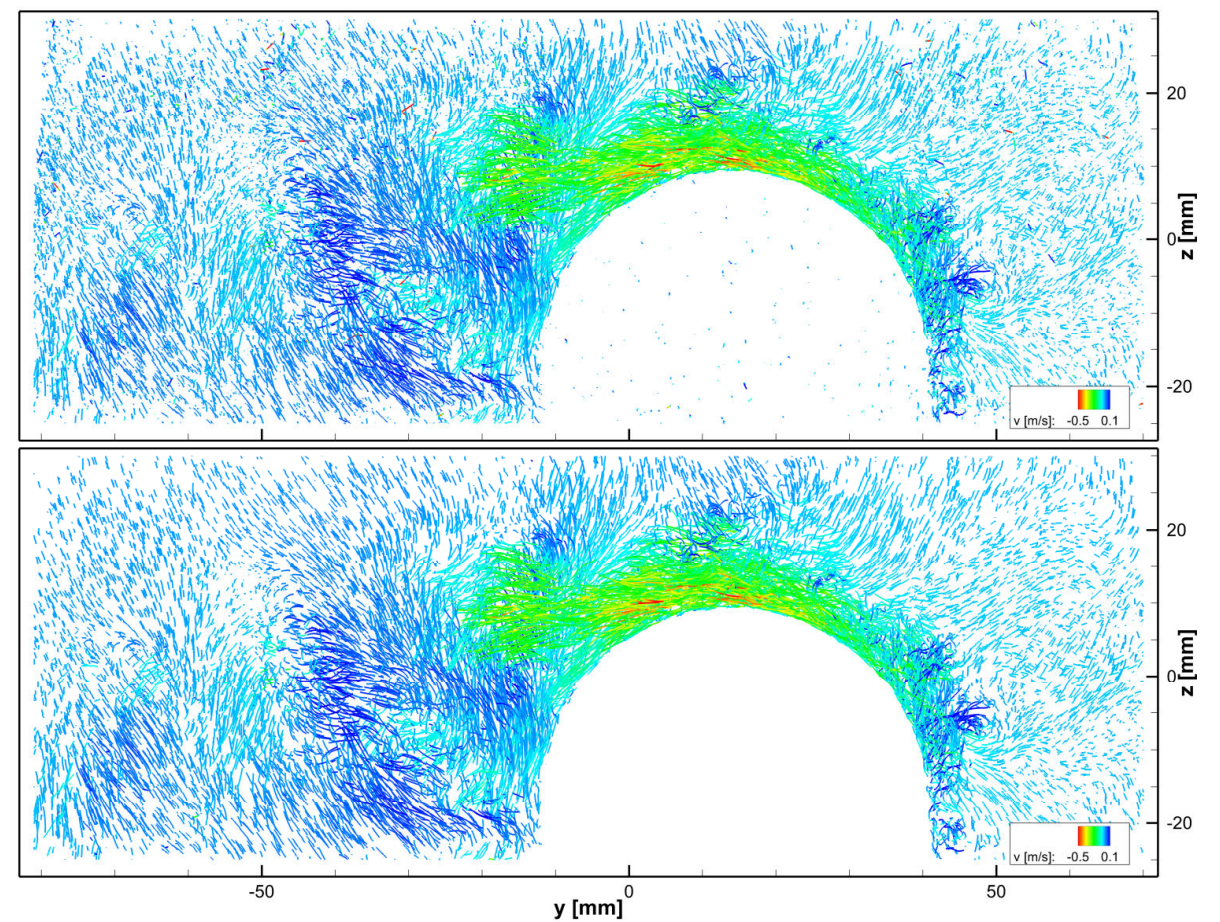

Fig. 7: STB processing (top) vs. VT-STB processing (bottom), showing tracks for 200 consecutive time-steps in a $10 \mathrm{~mm}$-thick slice in the $\mathrm{y}-\mathrm{z}$ plane, located as indicated in Fig. $3 \mathrm{c}$ ). 
These findings are supported by Fig. 7, which shows a 10 mm-thick slice in the y-z plane for both evaluations. Again it is obvious that VT-STB was able to efficiently suppress the generation of low-velocity ghost tracks and outliers, which are especially obvious in the outer regions and the cylinder for the STB evaluation.

Finally, Fig. 8 compares the two evaluations in a X-Z plane located on the upper edge of the measurement volume, above the jet (viewing from top). A vortical structure that is induced by the impingement of the jet and reaches up to the water surface is clearly visible in both reconstructions. The STB evaluation shows clear signs of 'line-of-sight'tracks, where particles follow the viewing direction of a singular camera, as their intensity is mostly supported by a peak on that specific camera, as argued in the introduction. The approximate camera positions in relation to the view in Fig. 8 can be taken from Fig. 2 a). Furthermore, it can be seen that some of the particles in the center of the vortex that are present for VT-STB are missing for the STB evaluation, which might be attributed to the lower choice of $T_{O}$ or the general lower reliability of STB in this case. The fact that the edges of the volume (where particles are not seen by all cameras) are not filled up for the STB case can be attributed to the shorter time-series that was evaluated $(1,000$ vs. 10,000 images). Such regions, where no new particles are triangulated have to be filled up by particles convecting from regions visible by more cameras. These missing regions also explain, why STB is tracking less particles, despite the non-negligible number of ghost particles.
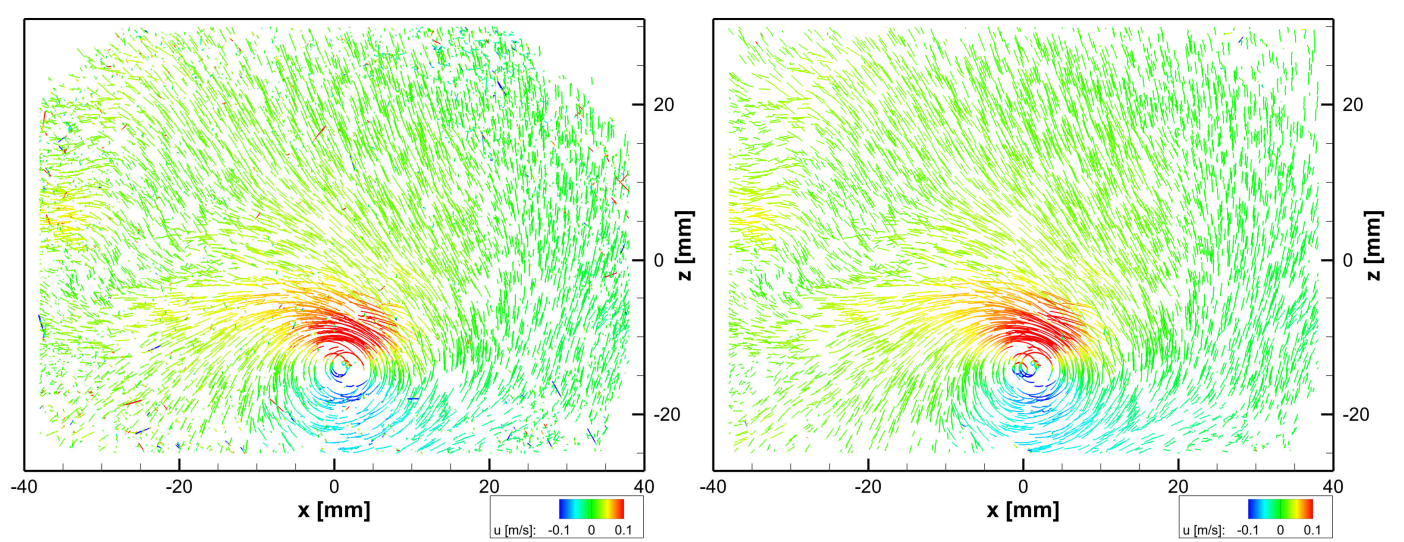

Fig. 8: STB processing (left) vs. VT-STB processing (right), showing tracks for 200 consecutive time-steps in a 10mm-thick slice in the $\mathrm{X}-\mathrm{z}$ plane, located as indicated in Fig. $3 \mathrm{~d}$ ).

It could be argued that using stricter parameters for normal STB might gain better results. While this is true in terms of obvious outliers, it has been found that reducing e.g. $R$ leads to a significant loss of high-velocity particles in the jet and the shear layer. Low-velocity ghost particles cannot be reduced by such measures.

Finally, it has to be stressed that the presented experimental case represents an extreme case in terms of velocity range and volume ratio of fast and (very) slow moving particles. For most experimental investigations, normal STB evaluations will show significantly less ghost particles, as such vast regions of slowly moving particles are not often found. However, we expect benefits of VT-STB processing in most flows, as a number of advantages (adjustable parameters for different velocity regimes, velocity-adapted filtering) still persist.

A direct comparison of reconstruction times was not conducted due to the different lengths of the used time-series. However, it can be stated that VT-STB uses only moderately more processing time. The first iterations are fast, as the time-separation is large. In the later iterations, the particles from the previous ones are fed, thereby limiting the amount of work done by IPR. In case the shaking of particles from previous iterations is deactivated further gains in evaluation speed are attained.

\section{Acknowledgements}

This work was supported by the Deutsche Forschungsgemeinschaft (DFG) through Grant No. SCHR 1165/5-1 as part of the Priority Programme on Turbulent Superstructures (DFG SPP 1881). We thank Janos Agocs, Reinhard Geisler, Tobias Kleindienst and Carsten Fuchs for their contributions to constructing the water tank and the optical set-up. We specially thank Kyung Chun Kim, Mirae Kim and Eunseop Yeom for their contributions to the layout and setup of the experiment. 


\section{References}

Bosbach J, Schanz D, Godbersen P, Schröder A (2019) Dense Lagrangian particle tracking of turbulent rayleigh bénard convection in a cylindrical sample using Shake-The-Box. 17th European Turbulence Conference (ETC 2019), 3.-6. Sept. 2019, Torino, Italy.

Gesemann S, Huhn F, Schanz D, Schroder A (2016) From noisy particle tracks to velocity, acceleration and pressure fields using B-splines and penalties. 18th Int. Symp. on Appl. of Laser and Imaging Tech. to Fluid Mech. Lisbon, Portugal, July $4-7$ 2016

Huhn, F., Schanz, D., Gesemann, S., Dierksheide, U., van de Meerendonk, R., \& Schröder, A. (2017). Large-scale volumetric flow measurement in a pure thermal plume by dense tracking of helium-filled soap bubbles. Exp. Fluids, 58(9), 116.

Jahn, T. Schanz, D., Schröder, A. (2021) Advanced Iterative Particle Reconstruction for Lagrangian Particle Tracking, submitted to Exp. Fluids

Kim, M., Kim, H. D., Yeom, E., \& Kim, K. C. (2018). Flow characteristics of three-dimensional curved wall jets on a cylinder. Journal of Fluids Engineering, 140(4), 041201.

Maas HG, Grün A, Papantoniou D (1993) Particle tracking in three dimensional turbulent flows—part I: photogrammetric determination of particle coordinates. Exp Fluids 15:133-146

Malik N, Dracos T, Papantoniou D (1993) Particle tracking in three dimensional turbulent flows - part II: particle tracking. Exp Fluids 15:279-294

Nishino K, Kasagi N, Hirata M (1989) Three-dimensional particle tracking velocimetry based on automated digital image processing. Trans ASME J Fluid Eng 111:384-390

Ouellette NT, Xu H, Bodenschatz E (2006) A quantitative study of three-dimensional Lagrangian particle tracking algorithms. Exp Fluids 40(2):301-313

Raffel, M., Willert, C. E., Scarano, F., Kähler, C. J., Wereley, S. T., \& Kompenhans, J. (2018). Particle image velocimetry: a practical guide. Springer.

Schanz D, Gesemann S, Schröder A, Wieneke B, Novara M (2013) Non-uniform optical transfer functions in particle imaging: calibration and application to tomographic reconstruction. Meas Sci Technol 24:024009

Schanz D, Gesemann S, Schröder A (2016) Shake-The-Box: Lagrangian particle tracking at high particle image densities. Exp Fluids 57(5), 70.

Stasicki B, Schroder A, Boden F, Ludwikowski K (2017) High-power LED light sources for optical measurement systems operated in continuous and overdriven pulsed modes, Optical Measurement Systems for Industrial Inspection X 10329, 103292

Wieneke B (2007) Volume self-calibration for 3D particle image velocimetry. Exp Fluids 45:549-556

Wieneke B (2013) Iterative reconstruction of volumetric particle distribution. Meas Sci Technol 24:024008 